\title{
TREE-LIKE CONTINUA AND CELLULARITY
}

\section{R. RICHARD SUMMERHILL}

ABSTRACr. In this paper the equivalence of tree-like and cellular is proved for 1-dimensional continua in $E^{n}$. More precisely, if $X$ is a tree-like continuum, then the collection of all embeddings $h: X \rightarrow E^{n}$, $n \geqq 3$, such that $h[X]$ is cellular in $E^{n}$ is a dense $G_{\delta}$-subset of the collection of all maps from $X$ into $E^{n}$. Conversely, if $X$ is a 1-dimensional cellular subset of $E^{n}$, then $X$ is a tree-like continuum.

1. Terminology. Throughout this paper a continuum will be a nondegenerate compact connected metric space and a covering will be a finite open covering. The symbol $\sim$ should be translated "homotopic to." If $X$ is a continuum and $\theta=\left\{O_{1}, \cdots, O_{m}\right\}$ is a covering of $X$, the mesh of $\theta$, denoted mesh $\theta$, is the maximum of the diameters of the elements of $\theta$. The nerve of $\theta$, denoted $\mathscr{N}(\theta)$, is the abstract complex consisting of those simplexes $\left(O_{i_{1}} \cdots O_{i_{j}}\right)$ such that $O_{i_{1}} \cap \cdots \cap O_{i_{j}} \neq \varnothing$. A continuum $X$ is tree-like if for each $\epsilon>0$ there exists a covering $\theta$ of $X$ such that mesh $\theta<\epsilon$ and $\Re(\theta)$ is a contractible 1-complex.

Let $X$ be a subset of a topological space $Y$ and let $n$ be a nonnegative integer. The statement that $X$ has property $n-U V$ means that for each open set $U$ containing $X$, there is an open set $V$ containing $X$ and contained in $U$ such that each singular $n$-sphere in $V$ is homotopic to 0 in $U . X$ has property $U V^{n}$ if it has property $i-U V$ for each $i \leqq n$ and $X$ has property $U V^{\omega}$ if it has property $i-U V$ for each nonnegative integer $i$. $X$ has property $U V^{\infty}$ if for each open set $U$ containing $X$, there is an open set $V$ containing $X$ and contained in $U$ such that $V$ is contractible in $U$. For a good discussion of the $U V$ properties the reader is referred to Armentrout [1].

A subset $X$ of $E^{n}$ is said to be cellular in $E^{n}$ if there is a sequence $C_{1}, C_{2}, \cdots$ of $n$-cells in $E^{n}$ such that

(1) for each positive integer $i, C_{i+1} \subset$ Int $C_{i}$, and

(2) $\bigcap_{i=1}^{\infty} C_{i}=X$.

This paper is devoted to studying the relationship between treelike, the $U V$-properties, and cellularity in Euclidean space. In $\$ 2$ we show that for 1-dimensional continua they are essentially the same and in $\$ 3$ we prove an embedding theorem for tree-like continua.

Received by the editors December 8, 1969.

AMS 1970 subject classifications. Primary 5450; Secondary 5420, 5425.

Key words and phrases. Cellularity, continua, dimension, tree-like, $U V$-properties. 
2. An equivalence theorem. In this section we shall show that a 1-dimensional continuum $X$ is tree-like if and only if the image of each embedding of $X$ into $E^{n}$ has property $U V^{\infty}$. This is equivalent to the statement that there is an embedding $h$ of $X$ into some Euclidean space such that $h[X]$ is cellular.

LEMma 1. Let $X$ be a continuum in $E^{n}, n \geqq 3$. If $X$ is 1-dimensional, then $X$ has property $i$ - $U V$ for $i=0,2,3, \cdots$. If $X$ is tree-like, then $X$ has property $U V^{\omega}$.

Proof. Let $U$ and $W$ be open subsets of $E^{n}$ such that $\bar{W}$ is compact and $X \subset W \subset \bar{W} \subset U$. There is a positive real number $\epsilon$ such that if $A$ is any subset of $U$ which meets $\bar{W}$ and has diameter less than $\epsilon$, then the convex hull of $A$ is contained in $U$.

Let $\mathcal{O}=\left\{O_{1}, \cdots, O_{m}\right\}$ be a covering of $X$ by open sets contained in $W$ such that mesh $\theta<\epsilon / 3$ and $\Re(\theta)$ is a 1-complex. If $X$ is tree-like then $\mathcal{O}$ may be chosen so that $\Re(\theta)$ is contractible. For each $i=1$, $\cdots, m$, let $p_{i}$ be a point of $O_{i}$ such that the set $\left\{p_{1}, \cdots, p_{m}\right\}$ is in general position in $E^{n}$. Since $n \geqq 3$, the collection $L$ consisting of vertices $p_{1}, \cdots, p_{m}$ and 1 -simplexes $\left(p_{i} p_{j}\right)$ such that $O_{i} \cap O_{j} \neq \varnothing$ is a subcomplex of $E^{n}$. The choice of $\epsilon$ implies that $L$ is contained in $U$. Moreover, $L$ is the image under a simplicial embedding of $\Re(\theta)$ into $E^{n}$ and therefore is contractible if $X$ is tree-like.

Let $V=\bigcup_{i=1}^{m} O_{i}$. Using the methods employed in [4, p. 69], there is a mapping $f$ from $V$ onto $L$ such that $O_{i}=f^{-1}\left[\mathrm{~s}^{\circ} \mathrm{t} p_{i}\right]$ (here $\mathrm{s}^{\circ} \mathrm{t} p_{i}$ denotes the open star of $p_{i}$ in $L$ ). Note that $f$ moves no point $x$ in $V$ more than $\epsilon$, for if $x \in O_{i}$, then $d(x, f(x)) \leqq d\left(x, p_{i}\right)+d\left(p_{i}, f(x)\right)<\epsilon / 3+2 \epsilon / 3$ $=\epsilon$.

Now let $S$ denote the standard $k$-dimensional sphere for some nonnegative integer $k$ and let $g: S \rightarrow V$ be a map. Then $f g$ maps $S$ into $L \subset U$ and $d(g(y), f g(y))<\epsilon$ for each $y \in S$. Thus $f g$ and $g$ are homotopic in $E^{n}$ by a homotopy which moves $f g(y)$ to $g(y)$ along a straight line segment of length less than $\epsilon$. In particular, the choice of $\epsilon$ implies that $f g$ and $g$ are homotopic in $U$. But $f g[S]$ is contained in $L$ and therefore, if $k \neq 1, f g \sim 0$ in $L \subset U$. If $X$ is tree-like, then $f g \sim 0$ in $L \subset U$ for all nonnegative integers $k$. Thus $g \sim f g \sim 0$ in $U$ for the desired cases.

The next lemma is proved by Case and Chamberlin in [2].

Lemma 2. A 1-dimensional continuum is tree-like if and only if each continuous map of $X$ into any linear graph is homotopic to 0.

Lemma 3. If $X$ is a 1-dimensional continuum in $E^{n}$ having property $U V^{\infty}$, then $X$ is tree-like. 
Proof. Let $g: X \rightarrow K$ be a map from $X$ into a linear graph $K$. Since $g$ is homotopic to a map from $X$ onto a subcomplex of $K$, there is no loss of generality in assuming that $g$ is onto. Let $p_{1}, \cdots, p_{m}$ be the vertices of $K$ and for each $i=1, \cdots, m$, let $O_{i}=g^{-1}\left[s^{\circ} t p_{i}\right]$. Then $\mathcal{O}=\left\{O_{i}\right\}$ is a covering of $X$ and $\Re(\theta)$ is a 1-complex simplicially isomorphic to $K$. Let $U_{1}, \cdots, U_{m}$ be open subsets of $E^{n}$ such that $U_{i} \cap X=O_{i}$ for $i=1, \cdots, m$ and such that if $\mathcal{U}=\left\{U_{i}\right\}$, then $\mathscr{T}(\mathcal{U})$ is simplicially isomorphic to $\mathscr{N}(\theta)$. Let $U=\bigcup_{i=1}^{m} U_{i}$ and let $f: U \rightarrow K$ be a map such that $f^{-1}\left[\mathrm{~s}^{\circ} \mathrm{t} p_{i}\right]=U_{i}$ for $i=1, \cdots, m$. Note that for each $x \in X, f(x)$ and $g(x)$ lie in the same simplex of $K$ and therefore $g \sim f \mid X$ in $K$. We show $f \mid X$ is homotopic to 0 in $K$.

Now $X$ has property $U V^{\infty}$ in $E^{n}$ and $U$ is an open set containing $X$, so there is a homotopy $H^{\prime}: X \times[0,1] \rightarrow U$ such that $H^{\prime}(x, 0)=x$ and $H^{\prime}(x, 1)=x_{0}$ for some point $x_{0} \in U$. Define $H: X \times[0,1] \rightarrow K$ by $H=f \circ H^{\prime}$. Then $H(x, 0)=f(x)$ and $H(x, 1)=f\left(x_{0}\right)$.

THEOREM 1. If $X$ is a 1-dimensional continuum, then the following are equivalent:

(1) $X$ is tree-like,

(2) the image of each embedding of $X$ into $E^{n}$ has property 1-UV,

(3) the image of each embedding of $X$ into $E^{n}$ has property $U V^{\infty}$, and

(4) $X$ can be embedded as a cellular subset of some Euclidean space.

Proof. If $n \geqq 3$, then the implications $(1) \Rightarrow(2) \Rightarrow(3)$ follow directly from Lemma 1 . If $n<3$, then Lemma 5.1 of [1] applies.

If $h: X \rightarrow E^{n}$ is an embedding such that $h[X]$ has property $U V^{\infty}$, then McMillan [5] has shown that $h[X]$ is cellular in $E^{n+1}$. Thus (3) and (4) are equivalent (observing Lemma 5.1 of [1] again). The proof is then completed by applying Lemma 3.

The previous theorem and the results of [2] provide an interesting example concerning the $U V$-properties. Case and Chamberlin construct an example of a subset $X$ of $E^{3}$ which is not tree-like, but which has trivial Cech groups.

Corollary 1. There is a 1-dimensional continuum $X$ in $E^{3}$ which has trivial Čech homology groups, cohomology groups, and fundamental group, but not having property $U V^{\infty}$ in $E^{3}$.

2. Embeddings of tree-like continua in $E^{n}$. Throughout this section let $X$ be a fixed tree-like continuum. Let $F[X]$ denote the collection of all mappings from $X$ into $E^{n}$ with the compact open topology. Recall that $F[X]$ is a complete metric space (cf. [4]) with the usual sup metric.

Consider the following subsets of $F[X]$ : 


$$
\begin{aligned}
I[X] & =\{f \in F[X] \mid f \text { is an embedding }\}, \\
F_{c}[X] & =\left\{f \in F[X] \mid f[X] \text { is cellular in } E^{n}\right\}, \\
I_{c}[X] & =F_{c}[X] \cap I[X] .
\end{aligned}
$$

In this section we prove that if $n \geqq 3$, then $I_{c}[X]$ is a dense $G_{\delta}$-subset of $F[X]$. Note that if $n<3$, then $I_{c}[X]=I[X]$. We assume therefore that $n$ is a fixed integer $\geqq 3$.

If $\epsilon$ is a positive real number, an $\epsilon$-mapping $f: X \rightarrow E^{n}$ is an element of $F[X]$ such that for each $y \in f[X]$, the set $f^{-1}(y)$ has diameter less than $\epsilon$. For each $i=1,2, \cdots$, let $G_{i}$ be the subset of $F[X]$ consisting of all $1 / i$-mappings. The following result is proved in [4].

Lemma 4. For each positive integer $i, G_{i}$ is a dense open subset of $F[X]$. Moreover, $I[X]=\bigcap_{i=1}^{\infty} G_{i}$ is a dense $G_{\delta}$-subset of $F[X]$.

For each $i=1,2, \cdots$, let $C_{i}$ be the collection of all elements $f$ of $F[X]$ such that there is an $n$-cell $C$ in $E^{n}$ with $f[X] \subset$ Int $C \subset C$ $C N(f[X], 1 / i)$. (Here, the set $N(f[X], 1 / i)$ denotes the $1 / i$-neighborhood of $f[X]$ in $E^{n}$.) Clearly $F_{c}[X]=\bigcap_{i=1}^{\infty} C_{i}$. The following two lemmas show that each $C_{i}$ is a dense open subset of $F[X]$.

Lemma 5. $F_{c}[X]$ is dense in $F[X]$.

Proof. Let $g$ be an element of $F[X]$ and let $\epsilon$ be a positive real number. Lemma 4 implies that $X$ can be considered a subset of $E^{n}$ such that $g$ moves no point more than $\epsilon / 2$. Corresponding to $\epsilon / 2$, let $f$ and $L$ be as in the proof of Lemma 1 ; that is, $f$ maps $X$ onto the contractible 1 -complex $L$ in $E^{n}$ without moving points more than $\epsilon / 2$. Then $f$ and $g$ are within $\epsilon$ of each other and, since $L$ is collapsible, $f[X]$ is cellular in $E^{n}$.

LemMa 6. For each positive integer $i, C_{i}$ is an open subset of $F[X]$.

Proof. Suppose $f \in C_{i}$ and let $C$ be an $n$-cell in $E^{n}$ such that $f[X] \subset$ Int $C \subset C \subset N(f[X], 1 / i)$. Let

$$
\epsilon=\min \left\{d\left(f[X], E^{n}-\operatorname{Int} C\right), d\left(C, E^{n}-N(f[X], 1 / i)\right)\right\} .
$$

Since $\epsilon<1 / i$, any $\epsilon / 2$-approximation $g$ to $f$ will have the property that $g[X] \subset$ Int $C \subset C \subset N(g[X], 1 / i)$.

Theorem 2. If $n \geqq 3, I_{c}[X]$ is a dense $G_{\delta}$-subset of $F[X]$.

Proof. The previous lemmas imply that for each $i=1,2, \cdots$, both $G_{i}$ and $C_{i}$ are dense and open in $F[X]$. Thus $G_{i} \cap C_{i}$ is dense and open. By Theorem 2-79 of [3], the set $\bigcap_{i=1}^{\infty}\left(G_{i} \cap C_{i}\right)=I[X] \cap F_{c}[X]$ $=I_{c}[X]$ is a dense $G_{\delta}$-subset of $F[X]$. 
The following corollary is now obvious.

CoROllary 2. Let $X$ be a 1-dimension continuum in $E^{n}$ having property $U V^{\infty}$ and let $\epsilon$ be a positive real number. Then there is an embedding $h: X \rightarrow E^{n}$ such that $d(x, h(x))<\epsilon$ for each $x \in X$ and such that $h[X]$ is cellular in $E^{n}$.

\section{REFERENCES}

1. S. Armentrout, UV properties of compact sets, Trans. Amer. Math. Soc. 143 (1969), 487-498.

2. J. H. Case and R. E. Chamberlin, Characterizations of tree-like continua, Pacific J. Math. 10 (1960), 73-84. MR 22 \#1868.

3. J. G. Hocking and G. S. Young, Topology, Addison-Wesley, Reading, Mass., 1961. MR 23 \#A2857.

4. W. Hurewicz and H. Wallman, Dimension theory, Princeton Math. Series, vol. 4, Princeton Univ. Press, Princeton, N. J., 1941. MR 3, 312.

5. D. R. McMillan, Jr., A criterion for cellularity in a manifold, Ann. of Math. (2) 79 (1964), 327-337. MR 28 \#4528.

University of Missouri at Columbia, Columbia, Missouri 65201 